\title{
STRONGLY NONLINEAR WAVES IN 3D PHONONIC CRYSTALS
}

\author{
C. Daraio, V. Nesterenko, S. Jin \\ Materials Science and Engineering Program, University of California at San Diego, La Jolla CA 920, USA
}

\begin{abstract}
Three dimensional phononic crystal ("sonic vacuum" without prestress) was assembled from 137 vertical cavities arranged in hexagonal pattern in Silicone matrix filled with stainless steel spheres. This system has unique strongly nonlinear properties with respect to wave propagation inherited from nonlinear Hertz type elastic contact interaction. Trains of strongly nonlinear solitary waves excited by short duration impact were investigated. Solitary wave with speed below sound speed in the air and reflection from the boundary of two "sonic vacuums" were detected.
\end{abstract}

\section{INTRODUCTION}

Linear elastic phononic crystals are materials with a periodic structure causing acoustic band gap $[1,2]$. An approach for modeling of compressional waves in weakly nonlinear phononic materials "the phononic lattice solid with fluids (PLSF)" at the microscopic scale was proposed in [3]. This paper presents the results on wave dynamics in strongly nonlinear [4] phononic crystals based on granular chains in a Silicone elastomer or Teflon matrix.

\section{STRONGLY NONLINEAR WAVES}

Non-classical wave behavior appears if a chain of grains is "weakly" compressed [4]. The principal difference between this case and the "strongly" compressed chain is due to a lack of a small parameter with respect to a wave amplitude in the former case. Long wave equation for displacement $u$ in this case is:

$$
\begin{aligned}
& u_{t t}=-c^{2}\left\{\left(-u_{x}\right)^{\frac{3}{2}}+\frac{a^{2}}{10}\left[\left(-u_{x}\right)^{\frac{1}{4}}\left(\left(-u_{x}\right)^{\frac{5}{4}}\right){ }_{x x}\right\}_{x},\right. \\
& -u_{x}>0, \quad c^{2}=\frac{2 E}{\pi \rho_{0}\left(1-v^{2}\right)}, \quad c_{0}=\left(\frac{3}{2}\right)^{\frac{1}{2}} c \xi_{0}^{\frac{1}{4}} .
\end{aligned}
$$

Here $c$ is not a sound speed, instead $c_{0}$ is a sound speed corresponding to initial strain $\xi_{0}$.
This equation has no characteristic wave speed independent on amplitude (equation for general interaction law can be found in [4]). Despite its complex nature the equation has simple stationary solutions with unique properties. For example, supersonic solitary wave propagates with a speed $V_{s}$ depending on the ratio $\xi_{\mathrm{r}}$ of initial $\xi_{\mathrm{o}}$ and maximum $\xi_{\mathrm{m}}$ strains:

$$
V_{s}=\frac{c_{o}}{\left(\xi_{r}-1\right)}\left(\frac{4}{15}\left[3+2 \xi_{r}^{\frac{5}{2}}-5 \xi_{r}\right]\right)^{\frac{1}{2}} \text {. }
$$

This strongly nonlinear solitary wave is of a fundamental interest because Eq. 1 is more general than weakly nonlineaar $\mathrm{KdV}$ equation. In a system moving with a speed $V_{p}$, its periodic solution is represented by a sequence of humps $\left(\xi_{0}=0\right)[4]$ :

$$
\xi=\left(\frac{5 V_{p}^{2}}{4 c^{2}}\right)^{2} \cos ^{4}\left(\frac{\sqrt{10}}{5 a} x\right) .
$$

Solitary shape can be taken as one hump of periodic solution (it has only two harmonics) with finite length equal five particle diameters. This unique wave was observed in numerical calculations and detected in experiments [4]. Solitary wave can be considered as a quasiparticle with mass equal about 1.4 mass of grain in the chain and its speed $V_{s}$ has a nonlinear dependence on maximum strain $\xi_{\mathrm{m}}$ (or particle velocity $v_{\mathrm{m}}$ ): 
$V_{s}=\left(\frac{4}{5}\right)^{\frac{1}{2}} c \xi_{m}^{\frac{1}{4}}=\left(\frac{16}{25}\right)^{\frac{1}{5}} c^{\frac{4}{5}} \bigcup_{m}^{\frac{1}{5}}$

We may see that the speed of this wave can be infinitely small if the amplitude is small! It means that using this material as a matrix in NTPC (Nonlinear, Tunable Phononic Crystals) we can ensure infinite elastic contrast of components, important for monitoring of band gaps. At the same time speed of solitary waves can be considered as constant at any relatively narrow interval of amplitudes due to power law dependence with small exponent. These properties allow using NTPCs as effective delay lines with exceptionally low speed of signal. Simple estimation based on Eq. 4 shows that it is possible to create materials with impulse speed in the interval $10-100 \mathrm{~m} / \mathrm{s}$ corresponding to the amplitude of audible signal.

\section{EXPERIMENTAL RESULTS AND DISCUSSION}

We processed a 3-D phononic crystal (Fig. 1) based on a Silicone elastomer matrix filled with one-dimensional chains of steel spheres. We will present a results describing how these chains support waves of different amplitude and duration.

In experiments we measured the force between the bottom plate and the last particle in the chain resting on this plate (Fig. 2). Piezoelectric gauges were placed under the plates of different diameters allowing support one or seven chains. They were connected with a wave guide - a long steel rod with a length about $20 \mathrm{~cm}$ embedded into the massive steel block. Typical time of the electric circuit of

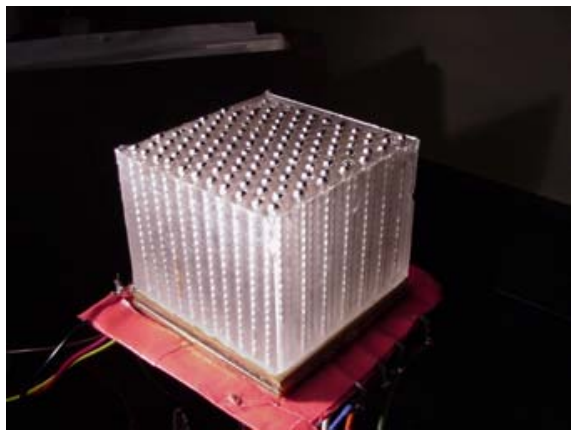

Figure 1. 3-D phononic crystal

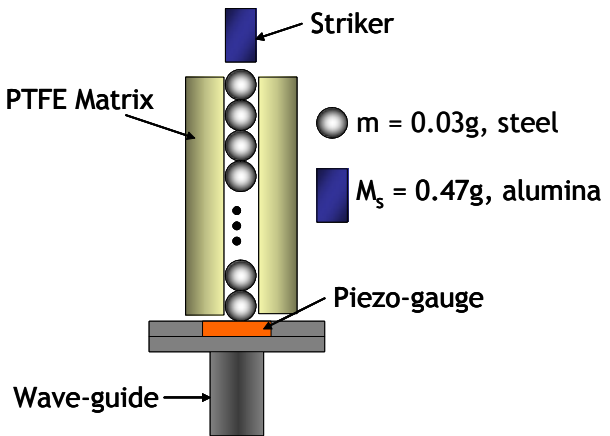

Figure 2. Set-up for testing of 1-D chain.

the gauge was $\mathrm{RC}=10^{3} \mu \mathrm{s}$ which was enough to ensure a good quality of signals with characteristic period up to $100 \mu \mathrm{s}$. The gauges were calibrated using impact with the parallel detection of acceleration.

One dimensional testing was performed using chains of balls placed in Teflon or Silicone elastomer matrixes to investigate how it may influence wave propagation in the chains (Fig. 3).

From Fig. 3 a remarkable feature of "sonic vacuum" is evident - very rapid decomposition of initial impulse on the distances comparable with the soliton width. In fact, the impulse is split after traveling only through 20 particles. This example also demonstrates that "short" duration impact on highly nonlinear ordered periodic systems (lattices) with weak dissipation may result in a chain of solitary waves instead of intuitively expected shock wave. Increase of the duration of impact results in shock wave with oscillatory structure where the leading pulse can be $\mathrm{KdV}$-type for weakly nonlinear chain or compacton-like for strongly nonlinear case [4].

This property of strongly nonlinear phononic crystal can be used for controlled impulse transformation in relatively short transmission lines. If chains of grains are placed into a polymer matrix the nonlinear elastic behavior is accompanied by strong dependence of electrical resistivity on local pressure [5]. This behavior can result in a new phenomena like train of locally conductive solitary waves.

Single solitary wave can be generated in the strongly nonlinear chains under impact of particles (pistons) with a mass equal or smaller than mass of particle in the chain [4]. 
PTFE Matrix
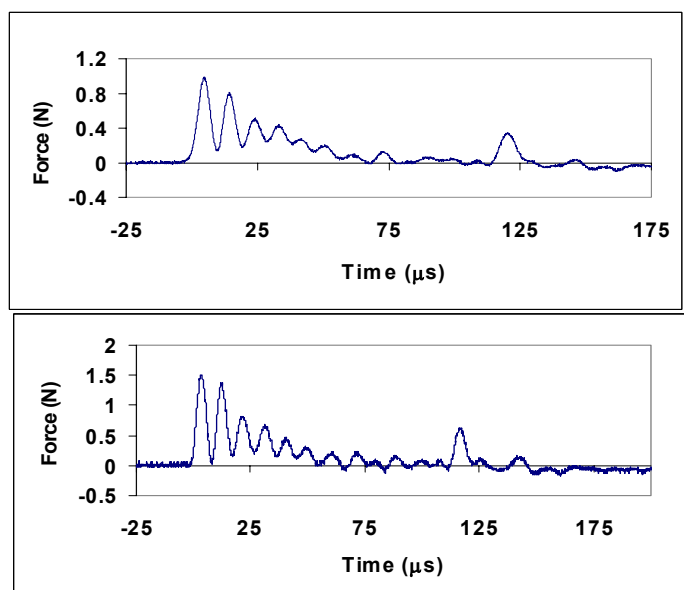

Silicon Matrix

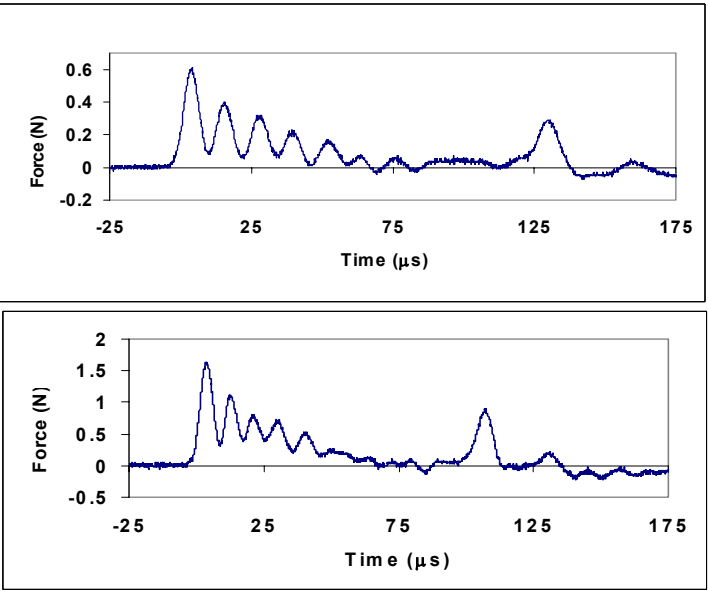

Figure 3. Impulses in a 1-D chains (20 steel particles with diameter $2 \mathrm{~mm}$ embedded into different matrixes) under identical loading using alumina striker (mass $0.47 \mathrm{~g}$, velocity $0.4 \mathrm{~m} / \mathrm{s}$ (top Figures) and $0.6 \mathrm{~m} / \mathrm{s}$ (bottom Figures).

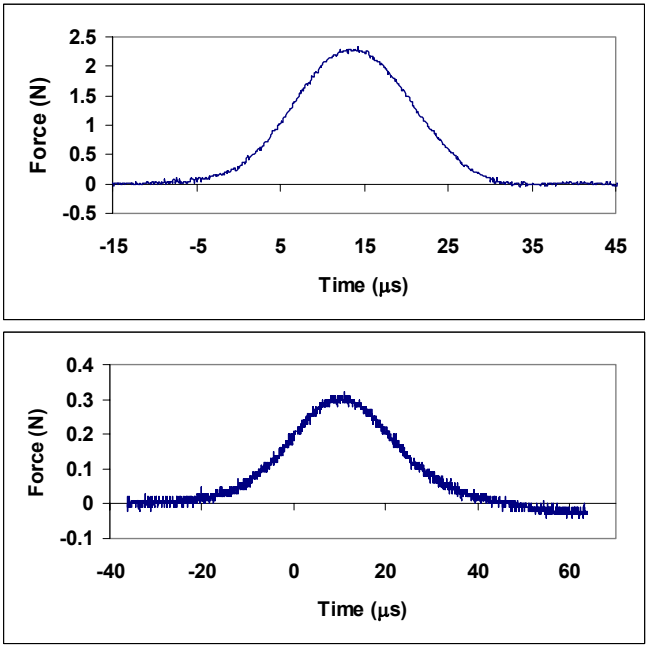

Figure 4. Solitary wave with amplitude significantly larger than gravitational prestress (top) and comparable to gravitational prestress.

The earlier experiments were conducted with amplitudes of solitary waves about $10 \mathrm{~N}$ (see references in [4]).

At the same time it is important to determine the possibility of these chains to support solitary waves with very small amplitude and small speed. For this reason we conducted experiments with loading of $5 \mathrm{~mm}$ diameter chains with 20 particles by impact of a single $2 \mathrm{~mm}$ diameter particle.

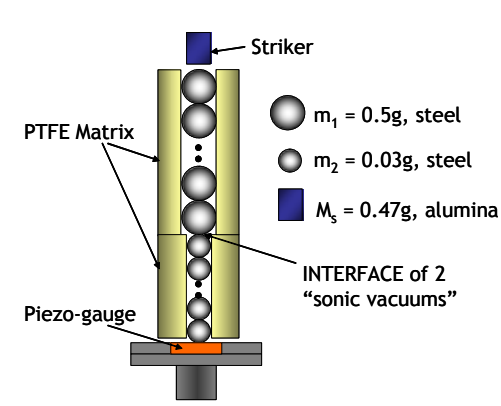

Figure 5. Set-up for the testing of reflection from interface of two "sonic vacuums".

The solitary signals were detected with small amplitudes $2.5 \mathrm{~N}$ and $0.3 \mathrm{~N}$ (Fig. 4). Based on the amplitudes (Eq. 4) a speed of solitary waves are equal $350 \mathrm{~m} / \mathrm{s}$ and $317 \mathrm{~m} / \mathrm{s}$ correspondingly, the latter is below sound speed in air.

Another type of testing was performed on interface between two "sonic vacuums" (Fig. 5) From a comparison of the signals (Fig.6) it is apparent that impulses marked by arrows correspond to the reflection of wave, originated on the bottom of the system, from the interface of two strongly nonlinear chains. Because the system is strongly nonlinear this reflection may not be described in terms of linear acoustics impedances.

Preliminary experiments with 3-D materials 
$10-10$

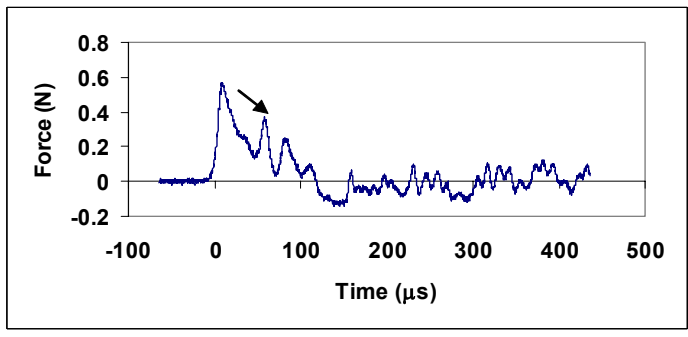

$5-10$

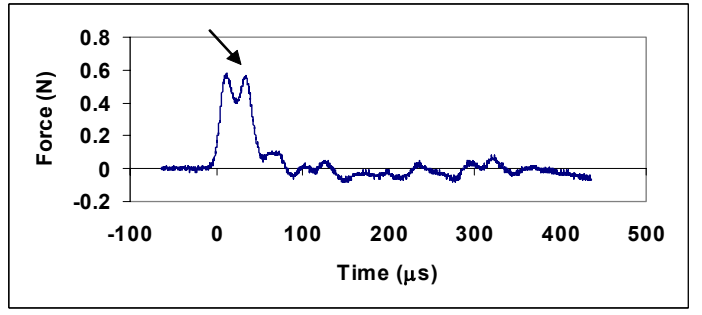

$10-20$

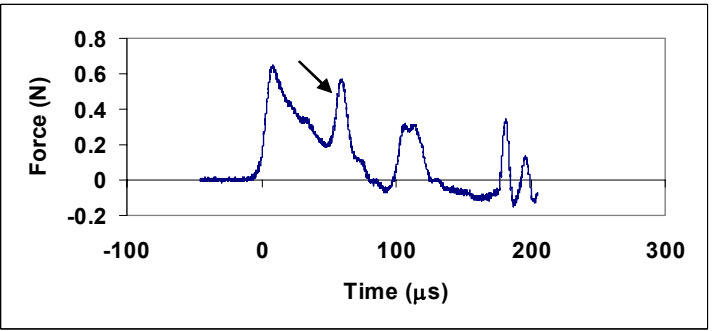

$5-20$

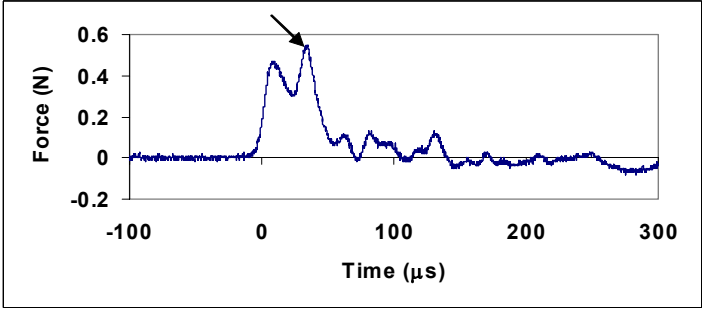

Figure 6. Wave reflection (shown by arrow) from the interface of strongly nonlinear chains.

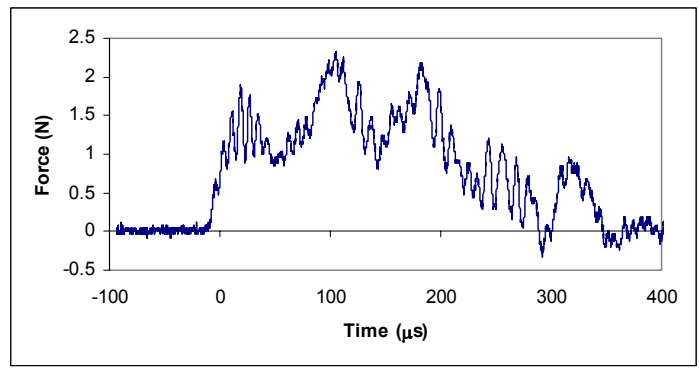

Figure 7. Wave in 7 central chains caused by impact of a steel ball $\left(\varnothing 10.5 \mathrm{~mm}\right.$, mass $\mathrm{M}_{\mathrm{s}}=5.3 \mathrm{~g}$ and $\left.\mathrm{u}_{\mathrm{s}}=0.4 \mathrm{~m} / \mathrm{s}\right)$ on a steel cover top plate with mass $7.65 \mathrm{~g}$.

were conducted using the central part of our phononic crystal (Fig. 1.) Seven chains were supported by the single plate placed on the top of piezogauge. The recorded signal (Fig. 7) corresponds to their collective action. It has oscillatory front and waves reflected from the cover plate back to the gauge are evident.

\section{CONCLUSIONS}

1-D and 3-D strongly nonlinear phononic crystals were assembled and investigated for different conditions of loading. Chain of solitary waves, reflection from the interface of two strongly nonlinear chains and single solitary waves with amplitude two orders of magnitude smaller than previously reported for chain of stainless steel spheres were observed. Small amplitude solitons broke "sound barrier" having speed of propagation below sound speed in air.

\section{ACKNOWLEDGEMENTS}

Funding was provided by the National Science Foundation, NSF grant \#0301322.

\section{REFERENCES}

1. Kushwaha, M.S., Classical band structure of periodic elastic composites. Int. J. of Modern Physics B, 1996. 10, 977.

2. Liu, Z., Zhang, X., Mao, Y., Zhu, Y., Yang, Z., Chan, C., and Sheng, P., Locally resonant sonic materials. Science, 2000. 289, 1734.

3. Lian-Jie, H. and Mora, P. The phononic lattice solid with fluids for modelling non-linear solid-fluid interactions. Geophysical Journal International, 1994. 117, 529.

4. Nesterenko, V.F., Dynamics of Heterogeneous Materials, Springer-Verlag, New York, 2001.

5. Jin, S., Tiefel, T.,Wolfe, R., Sherwood, R., and Mottine, J., Optically Transparent, Electrically Conductive Composite Medium. Science, 1992. 255, 446. 